\title{
Comparison of the Trabecular and Cortical Tissue Moduli from Human Iliac Crests
}

\author{
J. L. Kuhn, S. A. Goldstein, K. Choi, M. London, ${ }^{*}$ L. A. Feldkamp, and \\ L. S. Matthews \\ The Biomechanics, Trauma, and Sports Medicine Laboratory; The University of Michigan, Section of Orthopaedics, \\ Department of Surgery; Bioengineering Program, Ann Arbor; and *Physics Department, Ford Motor Company, \\ Dearborn, Michigan, U.S.A.
}

\begin{abstract}
Summary: The purpose of this study was to design a method to produce and test mechanically microspecimens of trabecular and cortical tissue from human iliac crests, and compare their measured moduli. Rectangular beam specimens were prepared on a low-speed diamond blade saw and a miniature milling machine. The final specimen dimensions ranged from $\sim 50-200 \mu \mathrm{m}$ for base and height. The modulus of each specimen was measured using three-point bending tests across a span length of $1.04 \mathrm{~mm}$ and performed at a constant rate of displacement. A subset of specimens was recovered for a radiographic estimation of degree of mineralization. The results showed the mean trabecular tissue modulus of all iliac crest specimens to be $3.81 \mathrm{GPa}$, whereas cortical tissue specimens averaged $4.89 \mathrm{GPa}$. This was a significant difference according to a two-way analysis of variance that controlled for differences between donors. No strong correlations were found between modulus and mineral density. Future investigations that consider other microstructural characteristics and their contributions to modulus, and specimen size effects, are indicated. Key Words: Trabecular tissue-Cortical tissue-Modulus-Bone microstructure.
\end{abstract}

An abundance of biomechanical research has been focused on the study of trabecular bone. Its mechanical function in load transmission, its remodeling potential, and its role as the host substance for artificial joints justify the intense interest in this tissue. Although two decades of experimentation and analysis have elucidated the nature of trabecular bone as a continuum material, the importance of trabecular architecture in determining macroscopic properties has been consistently emphasized. As such, more investigations have been di-

Presented in part at the 33rd Annual Meeting of the Orthopaedic Research Society, San Francisco, January 1987.

Address correspondence and reprint requests to Dr. J. L. Kuhn, The University of Michigan, Rm G-0161, 400 N. Ingalls, Ann Arbor, MI 48109-0486, U.S.A. rected toward an understanding of trabecular bone on a microstructural level.

Previous investigators have proposed structural models to describe the mechanical behavior of trabecular bone. An implicit assumption in these models has been that all bone tissue possesses the same physical properties on the microscopic level $(5,14,17,24)$. Consequently, trabecular elements have been assigned a cortical bone modulus of $\sim 13$ $\mathrm{GPa}$. Little data exist to support or refute this assumption. Townsend et al. (25) experimentally demonstrated in a buckling study of single human trabeculae that the modulus of trabecular tissue was very near that of cortical bone. Most recently, Ashman et al. (4) measured the elastic modulus of trabecular tissue from human and bovine distal femurs using ultrasound techniques. The average moduli 
were reported as $13 \mathrm{GPa}$ for human and $10.9 \mathrm{GPa}$ for bovine. Contradicting indirect evidence has also been published that suggests that the trabecular tissue modulus is considerably less than the cortical tissue modulus $(11,27)$. Weaver et al. (26) tested the microhardness of single trabeculae and compared it to that of the adjacent cortical bone, and found it to be "somewhat less." Pugh et al. (17) created a structural model of trabecular bone and assumed that the modulus of the trabecular tissue was 13.8 $\mathrm{GPa}$. As a result, it was necessary to reduce artificially the fraction of bone contained in the model so that the global mechanical stiffness corresponded to the measured stiffness of trabecular bone. This finding suggested that the modulus for trabecular tissue may be lower than that for cortical tissue. Ryan et al. (20) reported experimental modulus values for single bovine trabeculae as low as $758 \mathrm{MPa}$. Mente and Lewis (15) developed a combined experimental-finite element method to determine the modulus of single human trabeculae, and calculated an average elastic modulus of $5.3 \mathrm{GPa}$.

As a reasonable first step toward the ultimate objective of understanding trabecular bone micromechanics, the purpose of this study was to design a method to produce and mechanically test microspecimens of trabecular tissue and adjacent cortical tissue from human iliac crests, and compare their measured moduli.

\section{MATERIALS AND METHODS}

Three-point bending was chosen as the mechanical test, mainly due to the constraints of the specimen size required in testing single trabeculae.
Iliac crest trabecular and cortical specimens were excised from two fresh frozen cadavers: a healthy 23-year-old man (death by gunshot wound), and an active, healthy, 63-year-old man (death by sudden heart attack). Additional cortical specimens were produced from the femoral diaphysis of the 23year-old man. Specimens were also produced from the tibial diaphysis of a 45 -year-old woman (death by ovarian cancer) to test the specimen preparation technique. All bone material was obtained within 72 $h$ of death and fresh frozen at $-10^{\circ} \mathrm{C}$ in sealed bags until testing.

\section{Specimen Preparation}

Trabecular specimens were prepared by the following procedure. First, under constant irrigation, thin serial sections $(\sim 75-150 \mu \mathrm{m})$ from trabecular bone volumes were cut using a low-speed diamond blade saw (Model 11-1180, Isomet, Buehler, Lake Bluff, IL, U.S.A.). These sections were cleared of marrow so that all voids and tortuosities were clearly delineated. Bone segments deemed homogenous, continuous, and long enough to make good specimens were dissected from the trabecular sections.

Each bone segment was positioned across a 2$\mathrm{mm}$ wide slot in the bottom plate of a holding fixture and secured using a thin cover plate that contained a matching slot. The friction between plate contact surfaces was increased by sand paper (Fig. 1). The holding fixture was attached to the $x-y$ table of a specially designed miniature milling machine. With each trabecula stabilized in this manner, two sides were milled to a thickness of $\sim 50-200 \mu \mathrm{m}$. The cut-
FIG. 1. Schematic diagram of the holding fixture for the miniature milling machine: (a) top view, (b) side view. The bone segment is secured between two plates. Matching slots in both plates allow the 50-200 $\mu \mathrm{m}$ chrome cobalt drill bits to pass freely on either side of the bone segment.

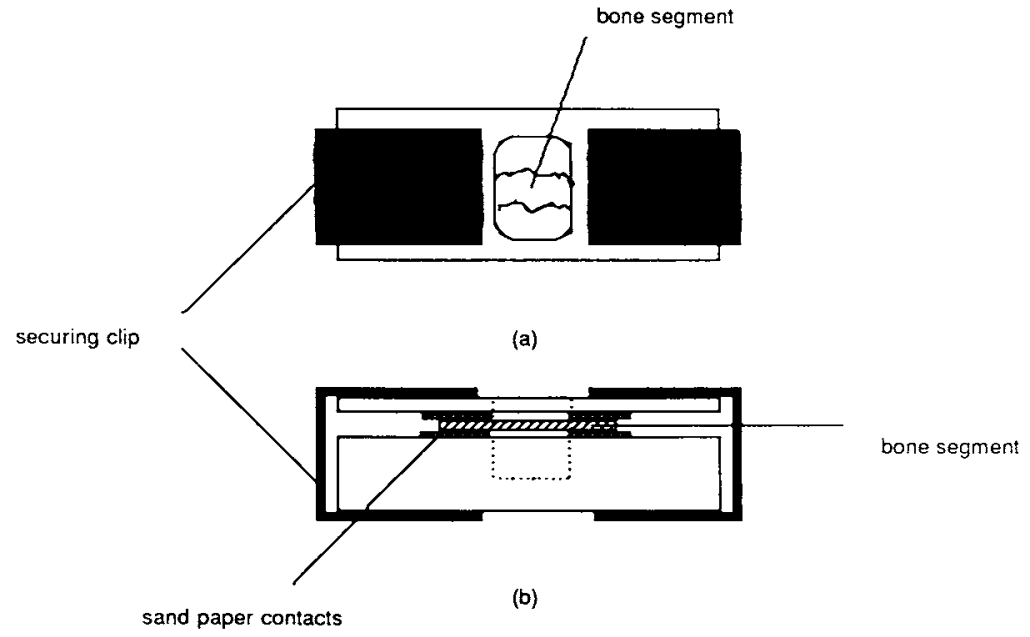

J Orthop Res, Vol. 7, No. 6, 1989 
ting tools consisted of a high-speed grinder (Model 604, Federal-Mogul Corporation, Chicago, IL, U.S.A.) and miniature chrome cobalt drill bits (\#3143, Vern Wheeler and Company, Livonia, MI, U.S.A.) of 50 and $200 \mu \mathrm{m}$ diameters. This milling procedure was conducted under a stereomicroscope at $\times 40$ magnification and produced rectangular beams of tissue. Specimens were kept wet at all times during this procedure by the stirring action of the drill bit in a water-filled well contained in the bottom plate of the holding fixture.

The majority of cortical specimens were produced according to this same procedure; however, a subset of specimens was prepared completely on the low-speed diamond blade saw. Each thin section of cortical tissue was sandwiched between two plates of plexiglass for support and cut sequentially at $75-150 \mu \mathrm{m}$ increments. This technique produced rectangular parallelopiped beam specimens in a relatively fast and efficient manner and also provided a second population of cortical specimens with which to statistically test the efficacy of the milling preparation technique.

To insure that microspecimens of cortical tissue were produced along the osteonal direction, each volume of iliac crest bone serving as a source for specimens was embedded in plastic for histological evaluation.

Specimen geometry was determined from digitized photomicrographs of specimen base and height taken at $\times 60$ magnification. The final dimensions of the rectangular beam specimens ranged on the average from 50 to $200 \mu \mathrm{m}$ for specimen base and height and 1,500 to $2,000 \mu \mathrm{m}$ for length (Fig. 2). Photographic exposure times of 2-3 min might have caused some drying of the specimens. However, every effort was made to keep the specimens moist during exposures by mounting specimens on wet filter paper. All specimens were immediately rewetted and retained frozen until testing.

It was decided that specimens with a base or height measurement $\geqslant 200 \mu \mathrm{m}$ might produce beams too short or too wide for classical beam theory to hold and, therefore, were thrown out. A total of 42 trabecular and 87 cortical specimens that were successfully prepared and mechanically tested is reported here.

\section{Mechanical Test}

An early 1950s Leitz microscope body and stage was converted to a three-point bending test apparatus. A highly sensitive and linear strain gauged load cell was placed in series with a platform that provided end supports spaced $1.04 \mathrm{~mm}$ apart for the test specimens. This assembly was attached to the vertically translating microscope stage. A stepper motor connected to the fine focal adjustment mechanism controlled this movement. A $160-\mu \mathrm{m}$-wide loading head was attached to the stationary base of the body tube. Beam displacement was measured by a linear variable differential transformer (Model 100 MHR, Schaevitz Engineering, Pensauken, NJ, U.S.A.) (Fig. 3). The compliance of the system was at least two orders of magnitude less than that measured for bone specimens.
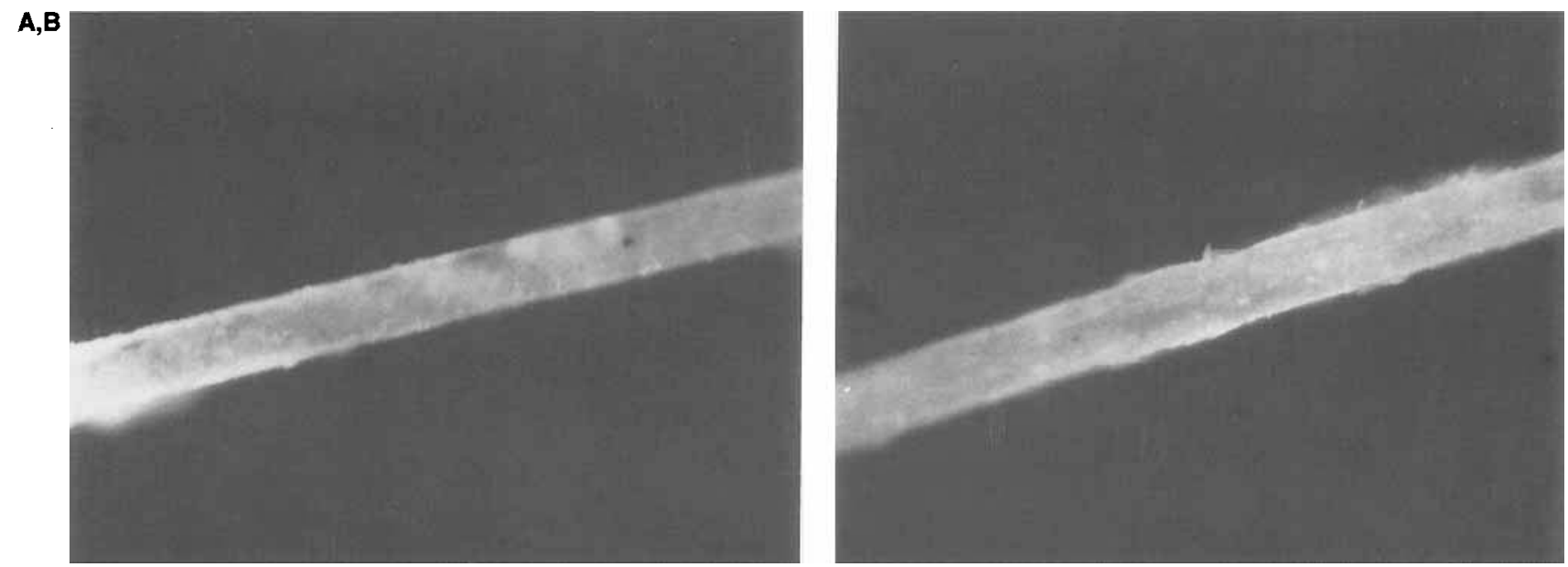

FIG. 2. Photographs taken at $\times 60$ magnification of a trabecular tissue specimen after milling. (A) The two sides of the beam created by the low-speed saw are shown. (B) The two sides of the beam created by the miniature milling machine are shown. 


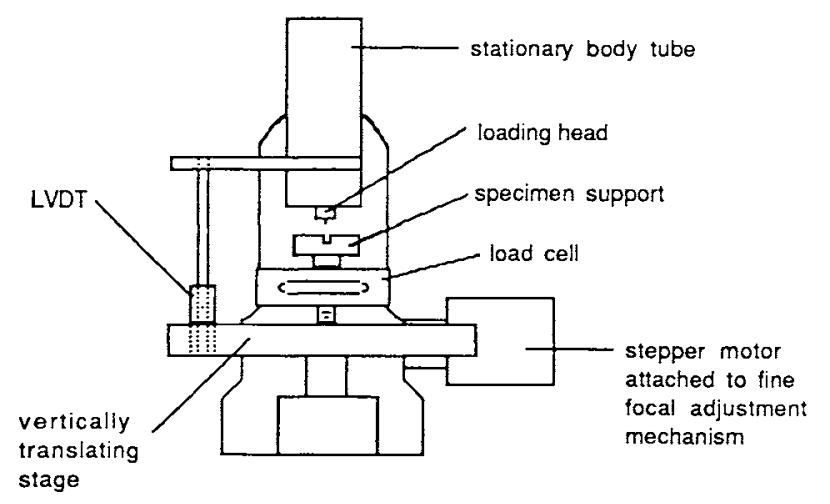

FIG. 3. Schematic diagram of the three-point bending test apparatus. A stepper motor attached to the fine focus knob controls the vertical motion of the stage. Specimens are positioned on the specimen support, and centrally loaded by a loading head attached to the stationary body tube of the converted microscope. Force and deflection data are acquired from a load cell in series with the specimen support stage, and a linear variable differential transformer (LVDT).

With the aid of a stereomicroscope, each specimen was placed across the supports and loaded at its midpoint at a constant displacement rate of 0.081 $\mathrm{mm} / \mathrm{s}$. The orientation of the specimen on the support was such that the Isomet cut surface was contacted by the loading head. The average heightto-base ratio was approximately $0.82: 1$, and the span length-to-thickness ratio had a mean of $\sim 12: 1$. Specimens were kept moist on saturated filter paper until positioned on the supports. Each test took $\sim 12-15$ s. Force and deflection data were acquired directly using an analog to digital converter and a Tektronix 4054 computer. Assuming isotropic, homogeneous, and linear elastic behavior, the following equation was used to calculate the modulus for each specimen (22):

$$
E=\frac{(P / y) L^{3}}{48 I}\left[1+2.85(h / L)^{2}-0.84(h / L)^{3}\right]
$$

where $E=$ tangent modulus, $P / y=$ slope of loaddeflection curve, $L=$ span length, $h=$ height, and $I=$ area moment of inertia.

This equation provides a correction factor for shear effects due to local perturbations of stress caused by the concentrated nature of the load. We felt that this was a reasonable analytic solution to use, particularly since the more classical equation without the correction factor is regularly used to calculate flexural moduli for plastics, ceramics, and laminated assemblies with height-to-base ratios of $0.15: 1$ to $2.0: 1$, and span length-to-thickness ratios of $8: 1$ to $16: 1$, a range not very different from that used in this study (ASTM D790-84, ASTM C674-80, and ASTM D1184-69.)

\section{Method for Estimating Degree of Mineralization}

Forty iliac crest trabecular specimens and 41 iliac crest cortical specimens were recovered after mechanical testing to estimate their degree of mineralization. An automated microradiographic technique, based on procedures developed for highresolution, three-dimensional computed tomography (9), was used to perform x-ray attenuation integral measurements along a large number of nearly parallel lines for each specimen. The most critical component was a microfocus $\mathrm{x}$-ray source (FeinFocus), which permits formation of highly magnified images of individual trabeculae. Each image possessed a magnification of $\sim 331$ pixels $/ \mathrm{mm}$. A quantity proportional to the average of the attenuation coefficient over a planar section was computed by integrating each attenuation profile (Fig. 4) and dividing by the relevant specimen crosssectional area. This quantity is assumed to be proportional to mineral density. An average mineral density was calculated from multiple profiles taken at different vertical positions along the length of the specimen near its fracture site. These calculated mineral densities reflected only relative differences between bone specimens since no attempt was made to calibrate the measures with phantoms of known density. However, relative differences should be sufficient to investigate the contribution of the degree of mineralization to modulus.

All recovered specimens were positioned freestanding supported by a clay base and scanned in a single session. Lined up in this manner, each spec-

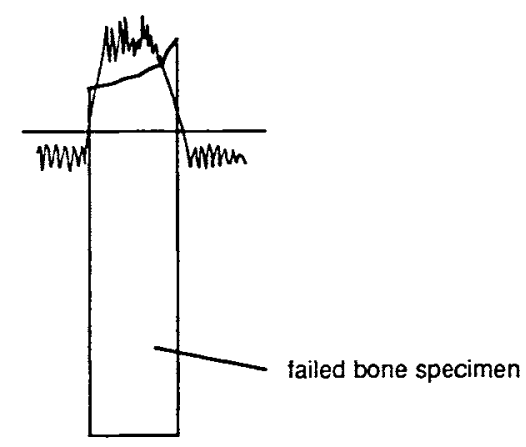

FIG. 4. Illustration of the method used to quantify a relative mineral density. Intensity profiles (variation in grey levels across the specimen) are integrated across each bone specimen image near the fracture site, and this value divided by the specimen's cross sectional area. 
imen was advanced into scanning position by use of a stepper motor. By eliminating the need for an embedding medium, the contrast between bone and background was enhanced, and the signal-to-noise ratio improved.

One important assumption in this method was that the calculated mineral densities were independent of specimen orientation during the scan (whether the specimen's base or height was parallel to the plane of the image intensifier). A small control experiment in which a vertically positioned bone specimen was multiply scanned at many different orientations showed that the variance in the calculated mineral density with different specimen orientation was small, with a $95 \%$ confidence interval of $\sim \pm 1 \%$ of the mean.

A second control experiment was performed to judge the reproducibility of the measurement. A subset of specimens was rescanned after shutting down the system, and reinitiating the start-up procedure, keeping all physical and $\mathrm{x}$-ray settings constant. The results showed a mean percent difference in the calculation of degree of mineralization to be $-0.96 \%$, an insignificant difference according to a pairwise $t$ test of these values $(p>0.5)$.

\section{RESULTS}

To evaluate the test apparatus, silicon carbide fibers with a diameter of $140 \mu \mathrm{m}$ (Textron Specialty Materials Division, Lowell, MA, U.S.A.) were used in control bending tests. The average measured modulus from tests of 20 specimens was $350.85 \mathrm{GPa}(\mathrm{SD}=17.84 \mathrm{GPa})$. This value falls within $10 \%$ of the manufacturer tested modulus, which has a statistical average of between $\sim 380$ and $414 \mathrm{GPa}$.

The efficacy of the milling preparation technique was proven by comparing the results from the 45 year-old tibial diaphyseal cortical specimens prepared in two different manners. Specimens prepared completely on the low-speed diamond blade saw $(n=15)$ averaged $5.09(\mathrm{SD}=3.3) \mathrm{GPa}$, and milled specimens $(n=14)$ exhibited an average modulus of 4.87 (SD = 2.4) GPa. A Student's $t$ test of this data showed the difference in modulus to be insignificant $(p>0.8)$, relieving our concern that the milling procedure might be introducing serious stress risers on the surface of the bone specimens.

A summary of the remaining mechanical test results is presented in Table 1. In general, the modu-
TABLE 1. Summary of results from mechanical tests ${ }^{a}$

\begin{tabular}{lcc}
\hline & $\begin{array}{c}\text { Trabecular tissue } \\
\text { modulus }\end{array}$ & $\begin{array}{c}\text { Cortical tissue } \\
\text { modulus }\end{array}$ \\
\hline 63-year-old iliac & $4.16(2.02)$ & $5.26(2.09)$ \\
crest & $n=29$ & $n=36$ \\
23-year-old iliac & $3.03(1.63)$ & $3.76(1.68)$ \\
crest & $n=13$ & $n=12$ \\
23-year-old femoral & & $4.59(1.83)$ \\
diaphysis & & $n=10$ \\
\hline
\end{tabular}

${ }^{a}$ All modulus values are reported in units of GPa. Standard deviations are shown in parentheses.

lus for all specimens ranged from $\sim 0.52$ to 13.84 $\mathrm{GPa}$.

The mean modulus of trabecular tissue from the 63-year-old iliac crest was $4.16 \mathrm{GPa}$, whereas the mean modulus of cortical tissue was $5.26 \mathrm{GPa}$. For the 23-year-old iliac crest, trabecular specimens averaged $3.03 \mathrm{GPa}$ and cortical specimens averaged $3.76 \mathrm{GPa}$. The average trabecular tissue modulus for all iliac crest specimens was $3.81 \mathrm{GPa}$, whereas the average cortical tissue modulus for all iliac crest specimens was $4.89 \mathrm{GPa}$. A two-way analysis of variance showed that (a) there was a significant difference between the 23- and 63-year-old iliac crest trabecular and cortical tissue modulus ( $p=0.006$ ) and (b) after controlling for the effect of different donors, the trabecular tissue modulus was significantly different from the cortical tissue modulus ( $p$ $=0.0505$ ).

A Student's $t$ test showed no significant difference in the cortical tissue modulus of the iliac crest and femoral diaphysis of the 23-year-old donor ( $p>$ 0.28 ). The mean modulus of the iliac crest specimens was $3.76 \mathrm{GPa}$, whereas the femoral diaphysis specimens averaged $4.59 \mathrm{GPa}$.

The specimens of this study exhibited a variation in mineral density near the fracture site, ranging between 0.19 and $0.595 \mathrm{U}$ with a mean of $0.384 \mathrm{U}$ and standard deviation of $0.08 \mathrm{U}$ (one outlier, greater than two standard deviations from the mean, was removed).

Using a Pearson product-moment correlation coefficient to indicate the strength of the linear relationship between mineral density and modulus, no strong relationships were found. Table 2 shows the correlation coefficients obtained for analyses (a) in which all specimens were included, (b) in which specimens were stratified by tissue type, and (c) in which specimens were stratified by both tissue type and donor. All correlation coefficients were $<0.5$. In two of these cases, the correlation coefficients 
TABLE 2. Correlation coefficients for modulus vs. mineral density

\begin{tabular}{cccccc}
\hline Group & $n$ & $r$ (linear) & $p$ & $r$ (log-log) & $p$ \\
\hline All & 81 & 0.28 & $<0.05$ & 0.295 & $<0.01$ \\
1,2 & 41 & 0.309 & $<0.05$ & 0.341 & $<0.05$ \\
3,4 & 40 & 0.136 & NS & 0.313 & $<0.05$ \\
1 & 34 & 0.305 & NS & 0.224 & NS \\
2 & 7 & 0.215 & NS & 0.548 & NS \\
3 & 27 & 0.499 & $<0.01$ & 0.63 & $<0.01$ \\
4 & 13 & 0.005 & NS & 0.06 & NS \\
\hline
\end{tabular}

Group $1=63$ year iliac crest, cortical,

Group $2=23$ year iliac crest, cortical.

Group $3=63$ year iliac crest, trabecular.

Group $4=23$ year iliac crest, trabecular.

$\mathrm{NS}=$ not significant, $p>0.05$.

were significant at a $5 \%$ level, and in one case the correlation coefficient was significant at a $1 \%$ level.

For completeness, an analysis of $\log$ modulus versus $\log$ mineral density was also performed to investigate the possibility of a power relationship between these two variables. These correlation coefficients are also displayed in Table 2. Correlation coefficients ranged from 0.06 to 0.63 , with two correlations significantly different from 0 at a $1 \%$ level, and two at a 5\% level. Figure 5 shows scatter plots of modulus versus mineral density and log modulus versus log mineral density.

\section{DISCUSSION AND CONCLUSIONS}

The goal of this study was two-fold. The first objective was to design a method to produce and mechanically test specimens of trabecular tissue, and the second was to use this method to compare the bending modulus of trabecular and cortical tissue in human iliac crests.

It was found that rectangular beam specimens
FIG. 5. Scatter plots of trabecular and cortical tissue modulus versus mineral density. Both linear and log plots are shown: group 163-year-old iliac crest, cortical; group 223-year-old iliac crest, cortical; group 363-year-old iliac crest, trabecular; and group 4-23-year-old iliac crest, trabecular. No strong relationships are revealed.
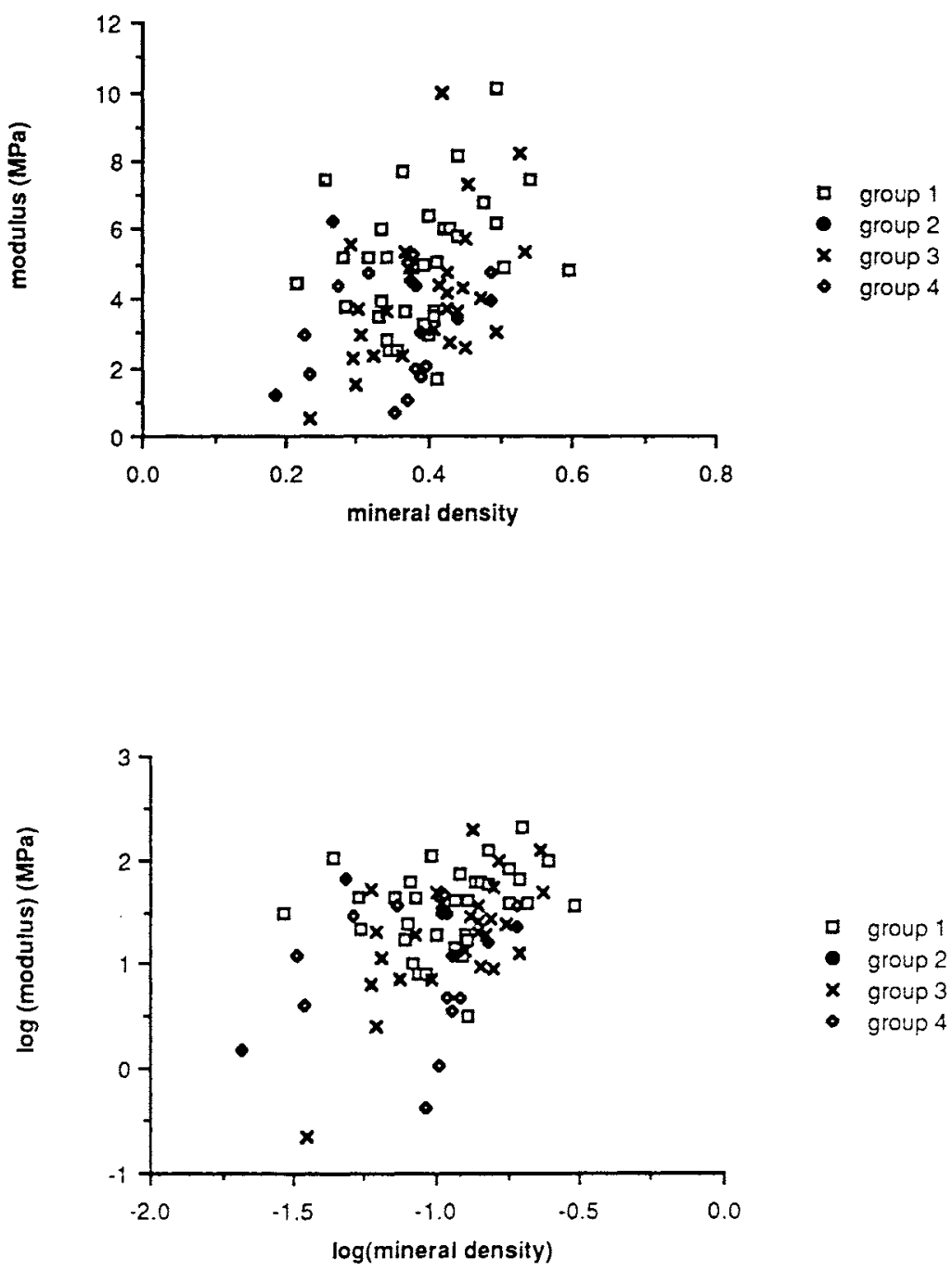
composed solely of trabecular tissue and suitable for three-point bending tests could be produced by our technique of (a) extracting individual bone spicules from thin sections of trabecular bone and (b) milling these bone segments into test specimens using specially designed holding fixtures, miniature chrome cobalt cutting tools, and a stereomicroscope. Although strict adherence to parallelism and polished specimen surfaces could not be optimally controlled, the statistical evaluation of the specimen preparation technique verified that serious stress risers were not created on the specimen surfaces during the milling operation.

The trabecular tissue specimens of this study displayed moduli in the range of $0.52-9.97 \mathrm{GPa}$, with a mean modulus of $3.81 \mathrm{GPa}$, falling into the range of values previously measured for trabecular tissue. This range is from $758 \mathrm{MPa}$ (20) to $13 \mathrm{GPa}$ (4). The discrepancy in values may be partly due to diverse experimental protocols. The mechanical tests that have been used to test trabecular tissue have included buckling (25), tension (20), a combined cantilever bending and finite element method (15), ultrasound techniques (4), and, in this investigation, three-point bending. Only this study has attempted to create specimens of regular geometry. In all cases, many simplifying assumptions were required to test mechanically microspecimens, in hopes of providing some initial perceptions of trabecular tissue mechanical behavior.

The iliac crest cortical tissue specimens demonstrated a range in modulus values of 1.19-11.25 $\mathrm{GPa}$, with a mean modulus of $4.89 \mathrm{GPa}, \sim 1.28$ times greater than the mean trabecular tissue modulus. Though the mean cortical tissue modulus of this study is in great contradiction to the widely accepted modulus values for large specimens of cortical bone of $15-18 \mathrm{GPa}(18,21)$, it is not extremely different than values measured for small specimens of cortical bone. Murray et al. (16) mechanically tested thin disks $(300 \mu \mathrm{m}$ to $2.5 \mathrm{~mm})$ of cortical bone from the distal metaphysis and proximal diaphysis of human tibiae, and found the mean elastic moduli to be 5.04 and $7.06 \mathrm{GPa}$, respectively. Even closer to the size scale of our cortical tissue specimens, Ascenzi and Bonucci (3) tested portions of longitudinally oriented human osteons in quasi-static tension. For wet specimens of various degrees of mineralization and collagen fiber orientation, the mean moduli ranged from 4.81 to 12.9 $\mathrm{GPa}$. Another study conducted on single human osteons by Frasca et al. (10) measured a dynamic
Young's modulus of $11.7 \mathrm{GPa}$ for 10 specimens from the tibia of a 12-year-old donor. Although this value is more than twice the mean value determined in this study, the authors stated that their experimental error was probably on the order of $25 \%$. Further, they discussed a growth rate of the elastic modulus with frequency, which can translate to a fractional decrease in modulus by as much as $50 \%$ with a decrease in frequency from 350 to $35 \mathrm{~Hz}(10)$. Therefore, a greater modulus might be expected from dynamic tension tests performed at a frequency of $1,000 \mathrm{~Hz}$ than from a quasistatic test.

Based on these comparisons, one further factor that may have contributed to our lower modulus values is a specimen size effect. In general, the references cited above seem to show decreasing moduli with decreasing specimen size. Few biomechanics studies have directly addressed this topic. Yang and Lakes (28) have studied human compact bone in quasistatic bending as a function of specimen size. They concluded that for a considerable range of specimen sizes, compact bone mechanics were consistent with micropolar elasticity rather than classical elasticity, and that, due to micropolar effects, stiffening of specimens occurs. It is difficult to determine how their results apply to this study, particularly since our calculations based on classical elasticity should have overpredicted the true modulus if our specimens indeed behaved according to micropolar elasticity and experienced a stiffening effect. More importantly, the range of specimen sizes tested in their study varied from $\sim 1.4$ to $5.2 \mathrm{~mm}$ in diameter. They calculated a characteristic length that corresponded to the diameter of osteons. Our specimens were on the order of 50-175 $\mu \mathrm{m}$, dimensions that are smaller than diameters of osteons. It may be that at this specimen size the structural element and characteristic length now corresponds to the lamellae or collagen fibers.

It may be more likely that a specimen size effect arises from stress concentrations produced by the ultrastructure of bone tissue or the "defects" in what we have assumed to be a homogeneous material. The lacunae represent locations of numerous stress risers, which may have trivial effects on a macrospecimen, but potentially large effects on a microspecimen. It may also be possible that local inhomogeneities produced by variations in the siteto-site degree of mineralization have a more severe effect on mechanical properties measured from microspecimens than from macrospecimens. Rice et al. (19) speculated that the greatly increased sur- 
face-to-volume ratio of microspecimens may increase the influence of surface imperfections, thereby resulting in lower modulus values. Recent work by Choi et al. strongly confirms this relationship (6). Using the same apparatus as this experiment, macrospecimens of cortical bone $(>500 \mu \mathrm{m})$ displayed an average modulus of $\sim 15 \mathrm{GPa}$. However, the modulus gradually decreased with specimen size in a manner strongly related $\left(r^{2}=0.84\right)$ to the surface-to-volume ratio, a relationship that may have implications for trabecular bone remodeling.

We feel that valid comparisons of trabecular and cortical tissue moduli can only be made if specimen dimensions are of the same order; otherwise, the effects of microstructure on micromechanical properties will not be similar for both tissues. Since the limiting factor in such an experiment is the size of continuous spicules within trabecular bone, we are left to accept whatever specimen size effect exists for cortical bone.

The results of this study demonstrated that after controlling for donor differences, the mean modulus of the trabecular tissue specimens tested was significantly lower than that of the cortical tissue specimens. However, the difference was much smaller than an order of magnitude, which might have been expected by some. Although the data also supported the fact that the difference in modulus due to donors was significant, no conclusions can or should be drawn concerning the age dependence of properties. It should be recognized that a very limited sample has been tested in this study and that until representative samples can be collected from multiple donors from many age groups, from both genders, and from multiple anatomic sites, no definitive or generalized statement can be made about the relationship between trabecular and cortical tissue moduli. Despite these limitations, these data do suggest that, in the iliac crest, the trabecular tissue modulus and cortical tissue modulus are on the same order of magnitude, but that the trabecular tissue modulus may be less in value than cortical by about $22 \%$.

The large variation in the modulus data was initially thought to be related to differences in degree of mineralization since such a relationship had been demonstrated by past studies performed on macroscopic specimens of cortical and trabecular bone $(7,8,12)$. It was interesting to find that in this study no strong correlations existed between mineral density and modulus. We hypothesize that the large standard deviations in the modulus data may be at- tributed to microstructural differences not examined in this preliminary study.

The extreme relevance of collagen fiber orientation and other microstructural characteristics to cortical bone mechanical properties has been established in earlier studies $(3,8,11,13,23)$. Amprino (1) found a direct correlation between cortical bone microhardness and degree of mineralization but found it to be certain, only if in tested bone areas all other microstructural characteristics were the same. Although not specifically tested by the authors, Ascenzi and Bonucci's results (3) seem to attest to the truth in Amprino's (1) conclusions. From their data, the effect of mineralization on the modulus of single osteons seems only apparent when the data are stratified by collagen fiber orientation or osteon type. Such differences in microstructure between specimens of this study might explain why low correlations between modulus and mineral density were obtained.

Sources of error that may have contributed to variations in the data include measurement errors in specimen geometry and differences in the actual strain rate of outside fibers.

Accurate measures of specimen base and height are especially critical in three-point bending tests because the calculation of the modulus is related to the third power of the specimen height and the first power of the specimen base. Using a linearized error propagation formula, we calculated that a $5 \%$ error in the geometry measurement would result in a $16 \%$ error in modulus. Since special care was taken to consider in the geometry measurement only those portions of the specimen that were clearly in focus, this estimate probably represents the worst case and errors were most likely $<10 \%$.

Although the displacement rate was constant at $81 \mu \mathrm{m} / \mathrm{s}$, the actual strain rate of outside specimen fibers depends on specimen height. Assuming linear elastic isotropy, the strain rate of the outside fibers varies linearly with respect to beam height. Given the range of specimen heights, $50-175 \mu \mathrm{m}$, estimated strain rates ranged from 0.22 to $0.79 \mathrm{~s}^{-1}$. Whether this is a sufficient difference to cause large variations in the modulus depends on the viscoelastic nature of trabecular and cortical tissue at this level. Little data have been published concerning the viscoelastic effects of microspecimens of bone tissue, although both Ascenzi et al. (2) and Frasca et al. (10) have conducted dynamic mechanical tests on single osteons.

Future experiments in which surface strains are 
concurrently measured with load and deflection may shed light on the validity of using classical beam theory as well as provide direct measurements of trabecular tissue failure strains. Lastly, the importance of microstructural interactions might be revealed by future studies incorporating polarized light analysis for examination of collagen fiber orientations, light and scanning electron microscopy for delineation of osteons, cement lines, and lacunae, and calibrated microradiographic techniques that show accurate point to point differences in mineral distribution.

It seems clear that the simple, original question relating trabecular and cortical tissue modulus is really a much more complicated question: that of the relationship between modulus and microstructure. Such an endeavor requires greater scrutiny than conducted here. The scope of such research was described by Sedlin and Hirsh in 1966 (21): "in order to account for the physical properties of wet bone on a microscopic basis, one must be prepared to reconstruct the three dimensional microanatomy of the specimen in the large majority of instances." With the present state of technology and the rate at which it is progressing, a study of such scope and magnitude may be less formidable than originally imagined.

Acknowledgment: We thank Textron Specialty Materials for their contribution of silicon carbide fiber samples, and George Lui, Lisa Degnore, Steve Stein, and Michael Herzig for their technical assistance. This work was supported by grants from the The Whitaker Foundation and the National Institutes of Health (AR 31793 and AR 34399).

\section{REFERENCES}

1. Amprino R: Investigations on some physical properties of bone tissue. Acta Anat 34:161-186, 1958

2. Ascenzi A, Benvenuti A: Mechanical hysteresis loops from single osteons: technical devices and preliminary results. $J$ Biomech 18:391-398, 1985

3. Ascenzi A, Bonucci E: The tensile properties of single osteons. Anat Rec 158:375-386, 1967

4. Ashman RB, Rho JY: Elastic modulus of trabecular bone material. J Biomech 21:177-181, 1988

5. Beaupre GS, Hayes WC: Finite element analysis of a threedimensional open-celled model for trabecular bone. $J$ Biomech Eng 107:249-256, 1985

6. Choi K, Kuhn JL, Ciarelli MJ, Goldstein SA: The elastic modulus of trabecular, subchondral, and cortical bone tis- sue. Presented at the 35th Annual Meeting of the Orthopaedic Research Society, February 1989.

7. Currey JD: The effect of strain rate, reconstruction and mineral content on some mechanical properties of bovine bone. J Biomech 8:81-86, 1975

8. Evans FG: Relations between the microscopic structure and tensile strength of human bone. Acta Anat 35:285-301, 1958

9. Feldkamp LA, Jesion G: 3D x-ray computed tomography. In: Review of Progress in Quantitative Nondestructive Evaluation, Vol 5A, ed by DO Thompson and DE Chimenti. New York, Plenum Press, 1986

10. Frasca P, Jacyna G, Harper R, Katz JL: Strain dependence of dynamic Young's modulus for human single osteons. $J$ Biomech 14:691-696, 1981

11. Gong JK, Arnold JS, Cohn SH: Composition of trabecular and cortical bone. Anat Rec 149:325-332, 1964

12. Kuhn JL, Goldstein SA, Ciarelli MJ, Matthews LS: The limitations of canine trabecular bone as a model for human: a biomechanical study. $J$ Biomech 22:95-107, 1989

13. Maj G, Toajari E: A resistenza meccanica del tessuto osseo lamellare compatto misurata in varie direzioni. Boll Soc Ital Biol Sper 12:83-86, 1937

14. McElhaney J, Alem N, Roberts V: A porous block model for cancellous bones. ASME Publication No. 70-WA/BHF-2, 1970, pp 1-9

15. Mente PL, Lewis JL: Young's modulus of trabecular bone tissue. Trans Orthop Res Soc 12:49, 1987

16. Murray RP, Hayes WC, Edwards WT, Harry JD: Mechanical properties of the subchondral plate and the metaphyseal shell. Trans Orthop Res Soc 9:197, 1984

17. Pugh JW, Rose RM, Radin EL: A structural model for the mechanical behavior of trabecular bone. $J$ Biomech 6:657670,1973

18. Reilly DT, Burstein AH: The elastic and ultimate properties of compact bone tissue. $J$ Biomech 5:393-405, 1975

19. Rice JC, Cowin SC, Bowman JA: On the dependence of the elasticity and strength of cancellous bone on apparent density. $J$ Biomech $21: 155-168,1988$

20. Ryan SD, Williams JL: Tensile testing of individual bovine trabeculae. In: Proceedings of the Twelfth Annual Northeast Bio-engineering Conference, ed by SC Orphanoudakis, pp 35-38, 1986, extended abstract

21. Sedlin E, Hirsch C: Factors affecting the determination of the physical properties of femoral cortical bone. Acta Orthop Scand 37:29-48, 1966

22. Timoshenko SP, Goodier JN: Theory of Elasticity, Third Edition. New York, McGraw-Hill Company, 1987, pp 113122

23. Toajari E: Resistenza meccanica ed elasticita del tessuto osseo studiata in rappor to alla minuta struttura. Monit Zool Ital 48:148-154, 1938

24. Townsend PR, Raux P, Rose RM, Miegel RE, Radin EL: The distribution and anisotropy of the stiffness of cancellous bone in the human patella. $J$ Biomech 8:363-367, 1975

25. Townsend PR, Rose RM: Buckling studies of single human trabeculae. J Biomech 8:199-201, 1975

26. Weaver JK: The microscopic hardness of bone. J Bone Joint Surg $[A m]$ 48:273-288, 1966

27. Williams JL, Lewis JL: Properties and an anisotropic model of cancellous bone from the proximal tibial epiphysis. $J B i$ omech Eng 104:50-56, 1982

28. Yang JFC, Lakes RS: Experimental study of micropolar and couple stress elasticity in compact bone in bending. $J$ Biomech 15:91-98, 1982 